\title{
Dynamic dual-source computed tomography imaging for myocardial perfusion
}

\author{
Allen Li *, YH Chan, BE Wu, CS Lam
}

Hong Kong Med J 2019;25:76-7.e1-3

http://doi.org/10.12809/hkmj177100

A 54-year-old man who was an ex-smoker was admitted to Pok Oi Hospital in August 2015 with acute chest pain that was subsequently confirmed to be a non-ST elevation myocardial infarction. Echocardiogram revealed anterior wall hypokinesia. Computed tomography (CT) coronary angiography demonstrated chronic total occlusion of the right coronary artery and a $90 \%$ stenotic lesion in the proximal to mid left anterior descending artery that was deemed to be the culprit lesion. Percutaneous coronary intervention was subsequently performed with a drug-eluting stent deployed across the proximal to mid left anterior descending artery stenosis. Angiographic results were excellent.

At clinical follow-up, the patient complained of persistent chest discomfort. Repeated echocardiogram was unremarkable showing normal left ventricular function without any regional wall motion abnormality. A CT stress myocardial perfusion and viability study was requested to guide subsequent management. The study protocol included quantitative evaluation of myocardial perfusion with pharmacological stress using a dynamic approach, followed by a delayed scan for the presence or absence of late myocardial enhancement.

Adenosine stress myocardial perfusion study with colour-coded maps demonstrated perfusion defects in the apicoseptal segment, the midinferoseptal segment, and to a lesser extent the basal inferoseptal segment (Fig 1). For quantitative evaluation, the normal areas had a myocardial blood flow of approximately $128 \mathrm{~mL} / 100 \mathrm{~mL} / \mathrm{min}$, whereas areas with ischaemia had a flow of around $40 \mathrm{~mL} / 100 \mathrm{~mL} / \mathrm{min}$. No delayed enhancement of the corresponding segments was evident to suggest scarring due to prior myocardial infarction (Fig 2).

Eventually the patient underwent a percutaneous coronary intervention to the chronic total occlusion of the right coronary artery in 2016 via a combined radial and femoral arterial approach with successful stent deployment across the occluded segment. Final angiography showed excellent results with mild residual stenosis in patent ductus arteriosus ostium (Fig 3). To date, the patient remains symptomfree with improvements in both his exercise tolerance and mood, and psychiatric reports revealing reduced dosage of antidepressants.

\section{Discussion}

Various imaging modalities are available for stress myocardial perfusion assessment. ${ }^{1}$ The present case demonstrates how a state-of-the-art dynamic and quantitative assessment of myocardial perfusion

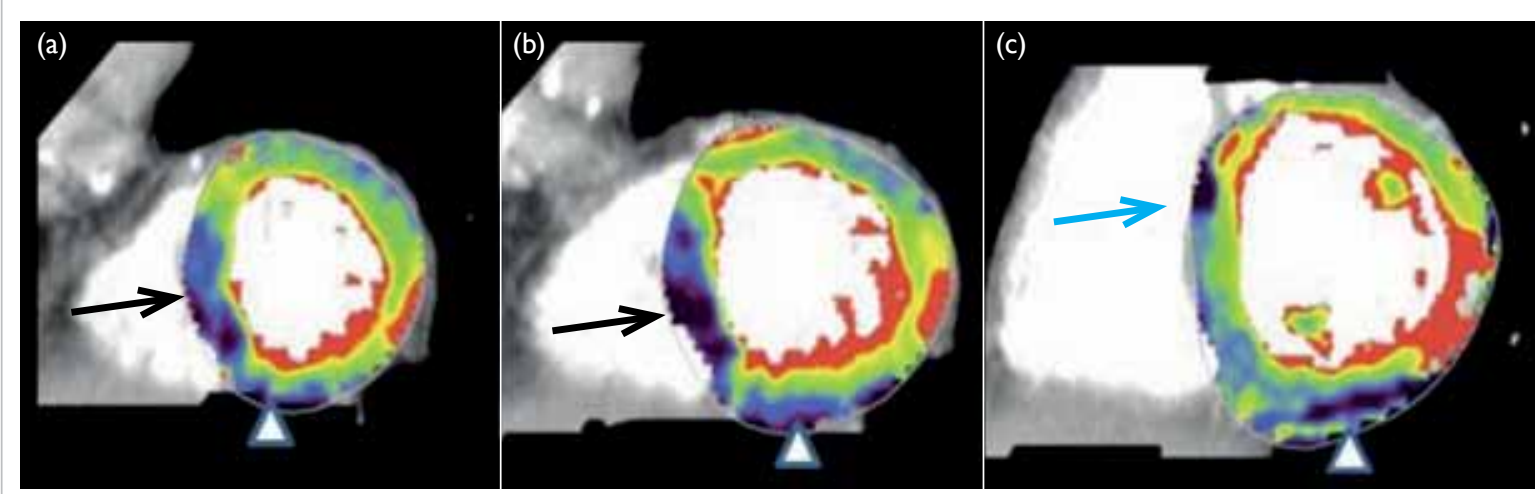

FIG I. Colour-coded maps of myocardial blood flow derived from stress dynamic computed tomography myocardial perfusion imaging (radiation dose about $8.5 \mathrm{mSv}$ ) showed significant perfusion hypoenhancement that involved (a) the apicoseptal segment, (b) the mid-inferoseptal segment, and to a lesser extent (c) the inferior aspect of the mid-basal anteroseptal segment (blue areas as indicated by the black arrows). Of note is the presence of artefacts at the subepicardial region of the mid-basal inferior segments (white arrowheads) and basal anteroseptal segment near the insertion point (blue arrow). Hibernating myocardium with reduced myocardial blood flow in the anterior segments from basal to apical levels 

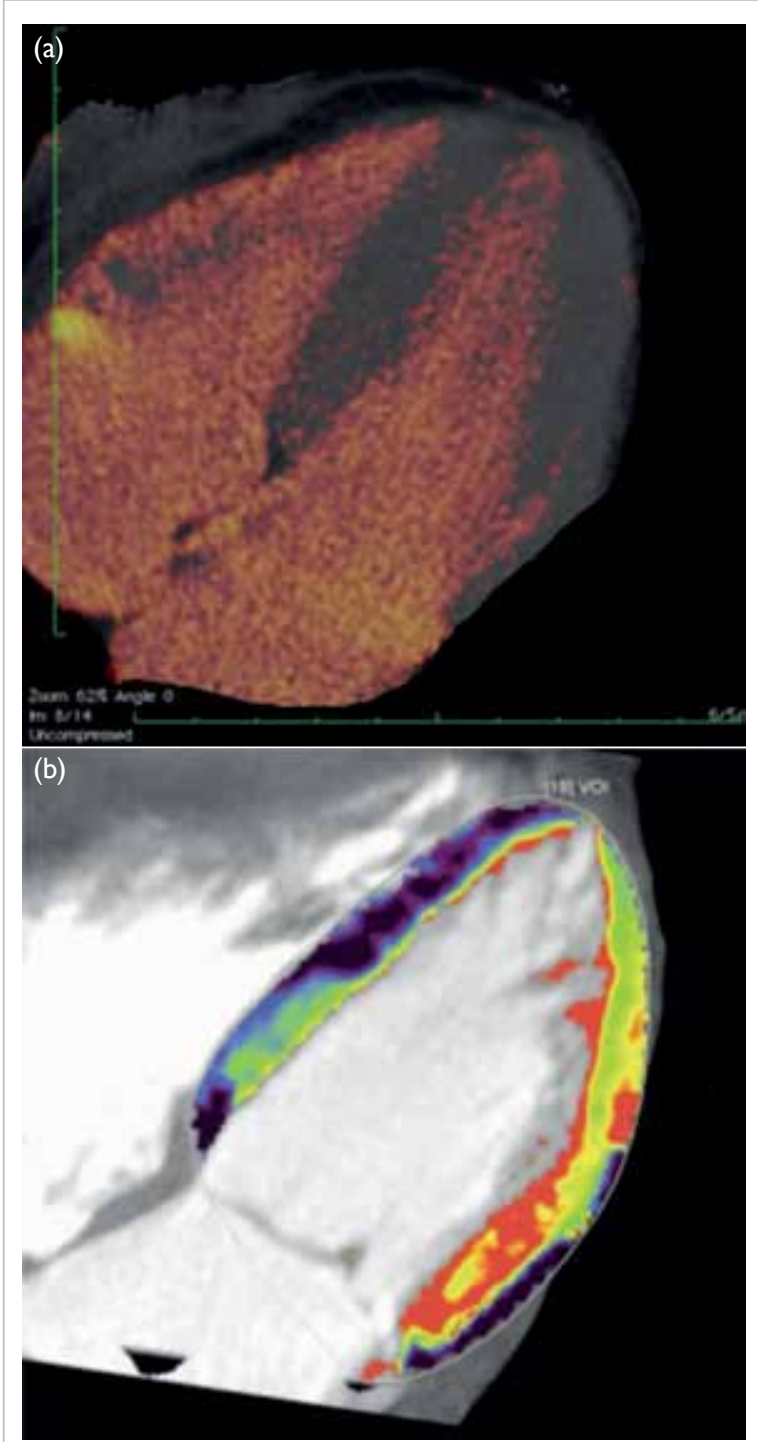

FIG 2. (a) Dual-energy computed tomography delayed enhancement showing no suspicious areas of late enhancement (radiation dose about $0.88 \mathrm{mSv}$ ). (b) Computed tomography myocardial perfusion study at the corresponding level demonstrates perfusion hypoenhancement (as indicated by the blue areas) of the inferoseptal segments
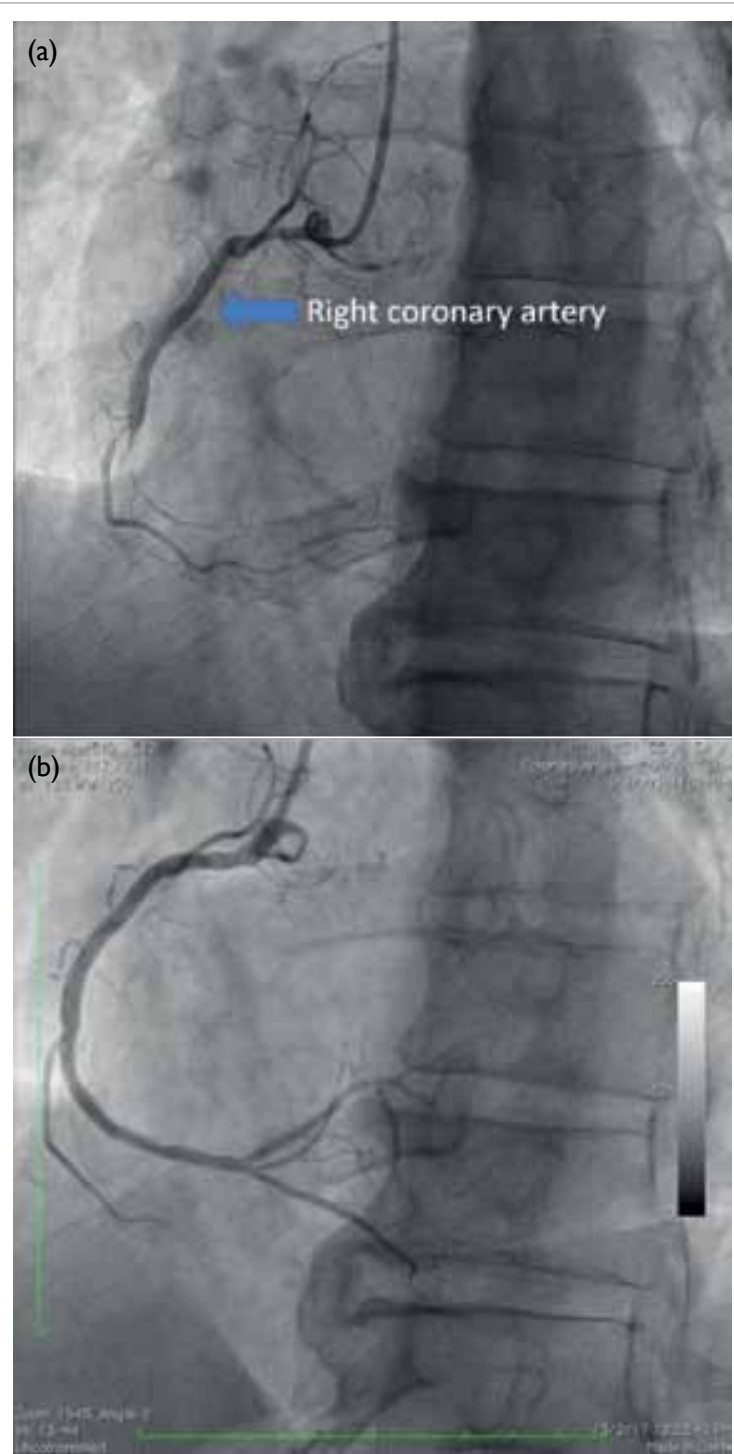

FIG 3. Coronary angiography demonstrating (a) chronic total occlusion of the right coronary artery with retrograde septal collaterals supplied from the left coronary system and (b) successful percutaneous coronary intervention with minimal residual stenosis of the patent ductus arteriosus ostium using a dual-source CT scanner enables detection of ischaemia along with viability assessment in a rapid and non-invasive fashion within an acceptable radiation dose. ${ }^{2}$

Conventional "static" CT myocardial imaging allows visual qualitative assessment of a single snapshot of myocardial iodine contrast attenuation that requires precise timing of the arrival of contrast to preserve diagnostic integrity. With a dual-source $\mathrm{CT}$, quantitative assessment of myocardial perfusion in multiple cardiac phases with precise anatomic localisation of the ischaemic area becomes possible. To date, many studies have assessed the reliability of dual-source multiple-detector $\mathrm{CT}$ in the dynamic and quantitative evaluation of myocardial perfusion..$^{2-5}$

Stress CT myocardial perfusion is emerging as a potentially promising non-invasive technique to detect myocardial ischaemia both qualitatively and quantitatively. With new-generation multipledetector CT scanners, a one-stop non-invasive comprehensive evaluation of the heart including the coronary artery, ventricular function, myocardial perfusion, and viability is possible. Stress CT myocardial perfusion provides incremental benefit to standard coronary CT angiography, particularly for intermediate coronary lesions. ${ }^{2}$ 


\section{Author contributions}

All authors had full access to the data, contributed to the study, approved the final version for publication, and take responsibility for its accuracy and integrity.

Concept or design: All authors.

Acquisition of data: A Li, YH Chan.

Analysis of data: A Li, YH Chan.

Drafting of manuscript: A Li, YH Chan.

Critical revision for important intellectual content: All authors.

\section{Conflicts of interest}

All authors have disclosed no conflicts of interest.

\section{Funding/support}

This research received no specific grant from any funding agency in the public, commercial, or not-for-profit sectors.

${ }^{1}$ A Li *, MB, ChB, FHKAM (Radiology)

${ }^{2}$ YH Chan, MB, BS, FHKAM (Medicine)

${ }^{3}$ BE Wu, MB, BS, FHKAM (Medicine)

${ }^{2}$ CS Lam, MB, BS, FHKAM (Medicine)

${ }^{1}$ Department of Radiology and Nuclear Medicine, Tuen Mun Hospital, Tuen Mun, Hong Kong

${ }^{2}$ Department of Medicine and Geriatrics, Pok Oi Hospital, Yuen Long, Hong Kong
${ }^{3}$ Department of Medicine and Therapeutics, Prince of Wales Hospital, Shatin, Hong Kong

* Corresponding author: liallen@yahoo.com

\section{References}

1. Ko SM, Hwang HK, Kim SM, Cho IH. Multi-modality imaging for the assessment of myocardial perfusion with emphasis on stress perfusion CT and MR imaging. Int J Cardiovasc Imaging 2015;31(Suppl 1):1-21.

2. Rossi A, Dharampal A, Wragg A, et al. Diagnostic performance of hyperaemic myocardial blood flow index obtained by dynamic computed tomography: does it predict functionally significant coronary lesions? Eur Heart J Cardiovasc Imaging 2014;15:85-94.

3. Kim SM, Choi JH, Chang SA, Choe YH. Additional value of adenosine-stress dynamic CT myocardial perfusion imaging in the reclassification of severity of coronary artery stenosis at coronary CT angiography. Clin Radiol 2013;68:659-68.

4. Bamberg F, Becker A, Schwarz F, et al. Detection of hemodynamically significant coronary artery stenosis: incremental diagnostic value of dynamic CT-based myocardial perfusion imaging. Radiology 2011;260:689-98.

5. Varga-Szemes A, Meinel FG, De Cecco CN, Fuller SR, Bayer RR 2nd, Schoepf UJ. CT myocardial perfusion imaging. AJR Am J Roentgenol 2015;204:487-97. 